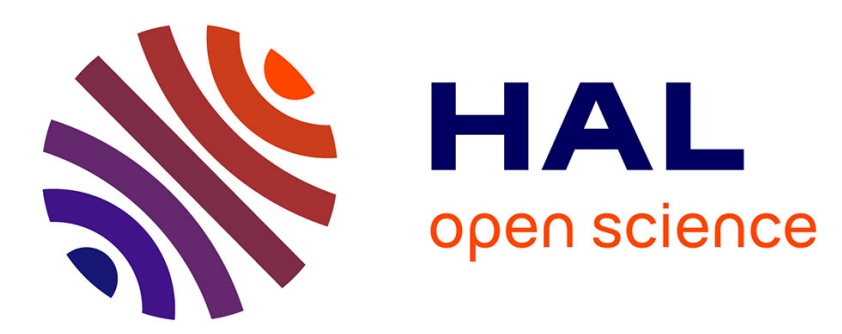

\title{
Bidimensional unconstrained optimization approach to EMD: an algorithm revealing skin perfusion alterations in pseudoxanthoma elasticum patients
}

Anne Humeau-Heurtier, Marcelo Colominas, Gastòn Schlotthauer, Maxime Etienne, Ludovic Martin, Pierre Abraham

\section{To cite this version:}

Anne Humeau-Heurtier, Marcelo Colominas, Gastòn Schlotthauer, Maxime Etienne, Ludovic Martin, et al.. Bidimensional unconstrained optimization approach to EMD: an algorithm revealing skin perfusion alterations in pseudoxanthoma elasticum patients. Computer Methods and Programs in Biomedicine, 2017, 140, pp.233-239. 10.1016/j.cmpb.2016.12.016 . hal-01430365

\section{HAL Id: hal-01430365 https://hal.science/hal-01430365}

Submitted on 21 Jun 2021

HAL is a multi-disciplinary open access archive for the deposit and dissemination of scientific research documents, whether they are published or not. The documents may come from teaching and research institutions in France or abroad, or from public or private research centers.
L'archive ouverte pluridisciplinaire HAL, est destinée au dépôt et à la diffusion de documents scientifiques de niveau recherche, publiés ou non, émanant des établissements d'enseignement et de recherche français ou étrangers, des laboratoires publics ou privés. 


\title{
Bidimensional unconstrained optimization approach to EMD: An algorithm revealing skin perfusion alterations in pseudoxanthoma elasticum patients
}

\author{
Anne Humeau-Heurtier ${ }^{\mathrm{a}, *}$, Marcelo A. Colominas ${ }^{\mathrm{b}, \mathrm{c}}$, Gastón Schlotthauer ${ }^{\mathrm{b}, \mathrm{c}, \mathrm{d}}$, \\ Maxime Etienne ${ }^{\mathrm{e}}$, Ludovic Martin ${ }^{\mathrm{e}}$, Pierre Abraham ${ }^{\mathrm{f}}$
}

\footnotetext{
${ }^{a}$ University of Angers, LARIS - Laboratoire Angevin de Recherche en Ingénierie des Systèmes, 62 avenue Notre-Dame du Lac, 49000 Angers, France

${ }^{\mathrm{b}}$ Laboratorio de Señales y Dinámicas no Lineales,Facultad de Ingeniería, Univ. Nacional de Entre Ríos, Argentina

c Consejo Nacional de Investigaciones Científicas y Técnicas (CONICET), Argentina

d Centro de Investigación y Transferencia de Entre Ríos (CITER). Argentina

e University of Angers, Angers Hospital, Department of Dermatology, UMR CNRS 6214-INSERM 1083, Angers, France

${ }^{\mathrm{f}}$ University of Angers, Angers Hospital, Laboratoire de Physiologie et d'Explorations Vasculaires, UMR CNRS 6214-INSERM 1083, Angers, France
}

\begin{abstract}
Background and Objective: Pseudoxanthoma elasticum (PXE) is an inherited and systemic metabolic disorder that affects the skin, leading among other things to a peau d'orange appearance. Unfortunately, PXE is still poorly understood and there is no existing therapy to treat the disease. Because the skin is the first organ to be affected in PXE, we propose herein a study of skin microvascular perfusion. By means of this analysis, our goal is to increase knowledge of PXE. Methods: For this purpose, microvascular data from patients suffering from PXE and from healthy control subjects were recorded using the laser speckle contrast imaging (LSCI) modality. These data were processed using the recent 2D version of the unconstrained optimization approach to empirical mode decomposition (UOA-EMD). Our work therefore corresponds to the first time this algorithm has been applied to biomedical data. Results: Our study shows that the 2D-UOA-EMD is able to reveal spatial patterns on local textures of LSCI data. Moreover, these spatial patterns differ between PXE patients and control subjects. Quantification measure of these spatial patterns reveals statistical significant differences between PXE and control subjects, in the neck $(p=0.0004)$ and in the back $(p=0.0052)$. Conclusions: For the first time, alterations in microvascular perfusion in PXE patients have been revealed. Our findings open new avenues for our understanding of pathophysiologic skin changes in PXE.
\end{abstract}

\section{Introduction}

Pseudoxanthoma elasticum (PXE) is a heritable systemic metabolic disorder that leads to ectopic calcification of soft connective tissue $[2,7,26]$. Alterations in the $A B C C 6$ gene have been associated with the disease (see, e.g., [3]). The organs that are primarily affected by PXE are the skin and the eyes [17]. Cutaneous lesions in PXE are asymptomatic yellow-white papules. These papules usually have a diameter of 2 to $5 \mathrm{~mm}$ and are "dome-shaped". The skin thus resembles an orange peel (peau d'orange), see Fig. 1. These papules are mainly found in the flexor areas: neck, axillae, and inguinal folds [17]. Moreover, the cardiovascular system may also be affected in PXE patients. Cardiovas-

\footnotetext{
* Corresponding author.

E-mail address: anne.humeau@univ-angers.fr (A. Humeau-Heurtier).
}

cular manifestations include, but are not limited to, arterial hypertension, angina pectoris, and reduced pulse amplitude. One study reported a relationship between the degree of cardiovascular involvement and severity of skin and mucous membrane lesions in PXE [50]. Recent studies estimate a prevalence of PXE as high as $1: 25,000[7,16,29,45]$.

Unfortunately, PXE is still a poorly understood condition and no definitive therapy exists. We noticed - in several instances - that the skin in PXE may be altered by presenting striking redness (socalled erythema). This prompted us to hypothesize that cutaneous PXE lesions could be associated with vascular changes. Hence our desire to study skin microvascular perfusion in PXE patients; such a study has not yet been conducted in the literature. The important questions to answer are: Is skin perfusion with lesions in PXE patients similar to that of control subjects? Is there any difference between skin perfusion presenting lesions and skin perfusion 


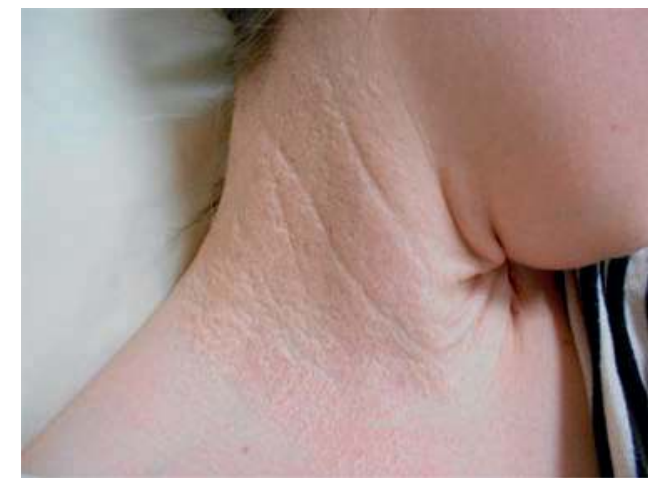

Fig. 1. "Orange peel" skin (peau d'orange) in the neck of a patient suffering from PXE.
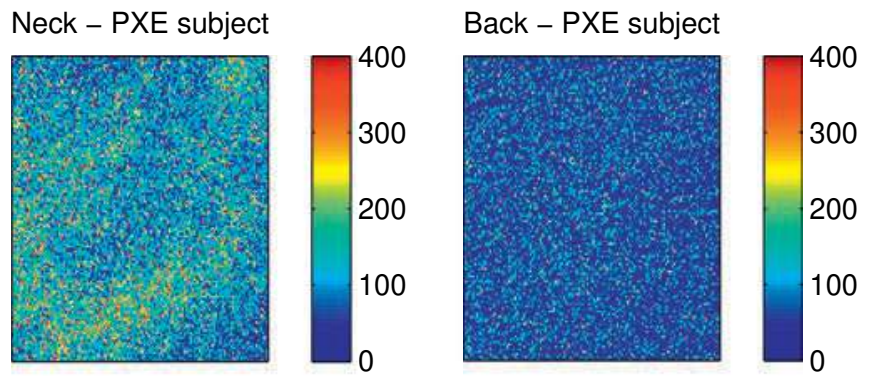

Neck - Healthy subject
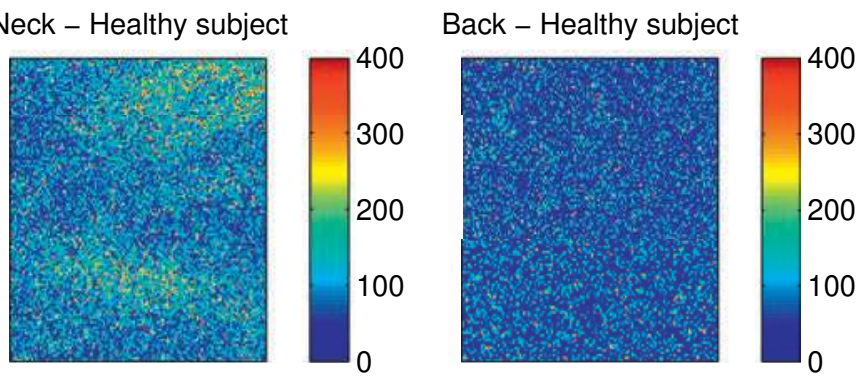

Fig. 2. Laser speckle contrast images recorded in the neck and back in a patient suffering from PXE and in a healthy subject. The size of the images is $60 \times 60$ pixels (see the text for details). The colorbar unit is $\mathrm{APU}_{L S C I}$. (For interpretation of the references to colour in this figure legend, the reader is referred to the web version of this article.)

without lesions? Does the systemic aspect in PXE pathology affect skin perfusion in regions where no papules are found? Finding answers to all of these questions would help in understanding the PXE disorder and might provide keys to possible therapy.

Skin microvascular perfusion can be assessed using the recently commercialized laser speckle contrast imaging (LSCI) modality [4$6,23]$. LSCI provides perfusion images with high temporal and spatial resolution and requires only low-cost devices [44]. Moreover, LSCI has the advantage of being contactless and the data are highly reproducible [46]. However, interpretation of LSCI data (nonstationary data) recorded from the skin is not an easy task since the images resemble noise (see examples in Fig. 2). It is therefore often necessary to use image/signal processing procedures to extract underlying physiological phenomena, which are useful for medical doctors $[22,24]$.

In the present work we propose a better understanding of the PXE pathophysiology through the processing of LSCI data recorded both in patients suffering from PXE and in control subjects. To this purpose, the task of image processing was performed by applying the new bidimensional version of the unconstrained optimization approach to EMD (2D-UOA-EMD, see below) algorithm to these LSCI data. Our study therefore presents two novelties: i) it is the first to propose an analysis of skin microvascular perfusion in PXE patients; ii) it is the first to propose a biomedical application of the recent 2D-UOA-EMD algorithm.

The paper is organized as follows. In Section 2, "Materials and Methods", we present the acquisition procedure as well as the 1D and $2 \mathrm{D}$ versions of the unconstrained optimization approach used to process the data. In Section 3, the results are detailed. They are discussed in Section 4. Conclusions are proposed in Section 5.

\section{Materials and methods}

\subsection{Acquisition procedure}

For this first application of the bidimensional version of the UOA-EMD, 2200 laser speckle contrast images were processed. The latter were recorded in 22 Caucasian subjects recruited from a reference center for PXE. The subjects were divided into two groups: eleven control subjects ( 7 men, 4 women, 31.09 years \pm 8.29 ) and eleven subjects with PXE (6 men, 5 women, 37.36 years \pm 7.70). The 22 subjects provided written, informed consent prior to participation and the study was carried out in accordance with the Declaration of Helsinki. The study was approved by the local ethics committee (composed of medical doctors, philosophers, psychologists, a jurist) under the number 2012/193. The control subjects were voluntary subjects working at the University hospital of Angers, France. They had normal skin (no papules). In the patients, the diagnosis of PXE was based on a combination of established criteria for indisputable PXE (clinically suggestive skin lesions, angioid streaks on fundoscopy, histological demonstration of fragmented and calcified elastic fibers on skin biopsy) as well as ABCC6 gene mutations.

For the perfusion image acquisition, the laser speckle contrast imager PeriCam PSI System (Perimed, Sweden) was used. The system consists of a linearly polarized laser emitting at a wavelength of $785 \mathrm{~nm}$ and using a camera with an exposure time of $6 \mathrm{~ms}$. The camera includes a polarizer aligned to remove specular reflection.

During acquisition, the subjects were placed supine in a quiet, temperature-controlled room with no air movement [34]. The distance from laser head to skin was set at $15 \mathrm{~cm}$ which gave images with a resolution of around $0.4 \mathrm{~mm}$ (see examples in Fig. 2). In each of the 22 subjects, two areas were imaged: the neck (area known for presenting papules in PXE patients) and the back (area known for absence of papules in PXE patients). LSCI perfusion data were acquired in laser speckle perfusion units $\left(\mathrm{APU}_{L S C I}\right.$, arbitrary units provided by the manufacturer), for $10 \mathrm{~s}$ in each site (neck and back) and stored with a sampling frequency of $5 \mathrm{~Hz}$ on a computer for off-line analysis. In each subject (11 healthy controls and 11 patients with PXE), fifty images recorded in the neck and fifty images recorded in the back were therefore taken into account. Before processing, the data were validated by experts (medical doctors recognized worldwide as experts in the field of LSCI or PXE - see our previous papers). In each of these images, a rectangular area was selected - mentioned below as a region of interest (ROI). The size of the ROI was approximately $576 \mathrm{~mm}^{2}$ (maximum area that can be chosen in the neck with the presence of papules; the same surface was therefore chosen in the back). In the back, the placement of the ROI was selected arbitrarily in each LSCI image. In the neck, the ROI was selected so as to show a region with papules. Each ROI was processed with the 2D-UOA-EMD framework (see below). In the healthy group, we therefore had $550 \mathrm{ROI}$ in the neck $(50 \times$ 11 ROI) and 550 ROI in the back; in the PXE group, we also had 550 ROI in the neck and 550 ROI in the back. A total of 2200 ROI were therefore processed. 


\subsection{Image processing framework}

\subsubsection{Empirical mode decomposition algorithm}

Empirical mode decomposition (EMD) leads to intrinsic mode functions (IMFs). The latter are functions directly conveying the amplitude and frequency modulations of the signal. An IMF satisfies two conditions [20]: (i) it contains the same number of extrema and zero crossings (or differs at most by one); (ii) the two IMF envelopes defined by its local maxima and local minima are symmetric with respect to zero. EMD relies on an iterative algorithm that uses a so-called sifting process to extract the IMFs [20]:

1. for a signal $X=h_{0}$, the mean $m_{0}$ of its upper and lower envelopes is determined from cubic-spline interpolation of local maxima and minima

2. $m_{0}$ is removed from the signal, giving the first component $h_{1}=$ $h_{0}-m_{0}$

3. the sifting process is iterated (here $h_{1}$ takes the place of $h_{0}$ ), leading to a new local mean $m_{1}$. Then $h_{2}$ is computed as $h_{2}=$ $h_{1}-m_{1}$

4. the procedure is repeated $k$ times until $h_{k}=h_{k-1}-m_{k-1}$ is an IMF, see the two conditions above (sifting process)

5. this first IMF is noted $c_{1}=h_{k}$ and contains the components with the highest local frequencies. We compute $r_{1}=X-c_{1}$ and then steps 1 to 4 are repeated on the residual signal $r_{1}$ leading to IMFs $c_{j}$ and residuals $r_{j}=r_{j-1}-c_{j}$, for $j$ varying from 1 to $n$

6 . the process stops when residual $r_{n}$ contains no more than three extrema.

EMD (and its variants) have been applied in a variety of fields (see, e.g., $[1,21,33,39]$ ). However, EMD has some drawbacks: mode mixing, or more generally, scale mixing [52,53]. Scale mixing corresponds to the presence of signals of widely disparate scales in a single IMF or as a signal of a given scale residing in several IMFs. Mode mixing is due to signal intermittency. Due to these drawbacks, the ensemble EMD (EEMD) was proposed in 2009 [52]. EEMD performs EMD over an ensemble of the signal under study plus Gaussian white noise. The 2D-version of EEMD has also been published in order to enable the processing of images [53]. However, EEMD itself has some drawbacks: residual noise is present in the reconstructed data and the mode mixing problem still resides in most cases for real applications (see, e.g., [49]). This is why other (numerous) algorithms have been proposed to overcome EMD and EEMD drawbacks: improved EMD [56], modified EEMD [58], complementary EEMD (CEEMD) [57], improved CEEMD [31], adaptively fast EEMD (AFEEMD) [55], partly EEMD (PEEMD) [59], complete EEMD with adaptive noise (CEEMDAN) [9,49], to cite only a few. In some cases, an $n$-dimensional version has also been proposed to process multidimensional data such as images [25].

\subsubsection{Unconstrained optimization approach to EMD}

Recently, Colominas et al. proposed an unconstrained optimization approach to EMD (UOA-EMD) [10]. The latter approach has the advantage of being simple and having an analytical solution. Moreover, due to the absence of envelope computation, drawbacks of EMD such as under- and overshoot are avoided. The UOAEMD algorithm relies on the following convex optimization problem (Tikhonov regularization) to find the local mean $a \in \mathbb{R}^{N}$ of a given signal $x \in \mathbb{R}^{N}[10]$ :

$$
\min _{a}\left\|P_{x}(x-a)\right\|_{2}^{2}+\lambda\|L a\|_{2}^{2},
$$

where matrix $P_{x} \in \mathbb{R}^{N \times N}$ is a linear operator, depending only on the locations of local extrema of $x$, which models the penalization imposed on the mode $d=x-a$ at each extrema, matrix $L \in \mathbb{R}^{N \times N}$ is a second-order difference matrix, $a$ and $x$ are considered column vectors, and $\|\cdot\|_{2}$ stands for the $\ell_{2}$-norm. This problem has a unique solution [10]:

$a^{*}=\left(P_{x}^{T} P_{x}+\lambda L^{T} L\right)^{-1} P_{x}^{T} P_{x} x$.

The matrix $P_{x}$ is a key element to finding the local extrema of $x$ : it has as many non-zero rows as local extrema of the signal $x$ [10]. Let $p_{l} \in \mathbb{R}^{N}$ be the $t_{l}$ th row of matrix $P_{x}$. It has non-zeros elements only on the $t_{l-1}, t_{l}, t_{l+1}$ th positions:

$p_{l}=\left[\ldots \frac{t_{l+1}-t_{l}}{t_{l+1}-t_{l-1}} \ldots 1 \ldots \frac{t_{l}-t_{l-1}}{t_{l+1}-t_{l-1}} \ldots\right]$.

Eq. (3) can therefore be used to construct matrix $P_{x}$. Using the latter, decomposition of signal $x$ becomes possible, as described in the algorithmic-based version shown in [8].

\subsubsection{Bidimensional extension of $U O A-E M D$}

A bidimensional extension of UOA-EMD (2D-UOA-EMD) has also been proposed recently [8]. When considering a function of two real variables $X=X(x, y)$, where $X \in \mathbb{R}^{N_{1} \times N_{2}}$ is the image, the bidimensional version is based on the $1 \mathrm{D}$ algorithmic-based version, where the $2 \mathrm{D}$ extension of the convex optimization problem is written as [8]

(P-2D) $\min _{\hat{a}}\left\|\hat{P}_{X}(\hat{x}-\hat{a})\right\|_{2}^{2}+\lambda\|\hat{L} \hat{a}\|_{2}^{2}$,

$\hat{x} \in \mathbb{R}^{N_{1} N_{2}}=\operatorname{vec}(X), \hat{a} \in \mathbb{R}^{N_{1} N_{2}}=\operatorname{vec}(A)\left(A \in \mathbb{R}^{N_{1} \times N_{2}}\right.$ is an approximation such that penalization on every local extrema of the mode $D=X-A$ is minimized). The matrix $\hat{P}_{X} \in \mathbb{R}^{N_{1} N_{2} \times N_{1} N_{2}}$ contains $\left(\operatorname{vec}\left(P_{l}\right)\right)^{T}$ as rows and $\hat{L} \in \mathbb{R}^{N_{1} N_{2} \times N_{1} N_{2}}$ contains $\left(\operatorname{vec}\left(L_{i, j}\right)\right)^{T}$ as rows. $P_{l} \in \mathbb{R}^{N_{1} \times N_{2}}$ is a matrix with $P_{l}\left(z_{i}\right)=c_{i}, i=1,2,3$, and the coefficients $c_{i}$ are those necessary to compare the function $D\left(z_{l}\right)$ with the plane intersecting $D\left(z_{i}\right)$ ( $z_{l}$ is a local maximum (resp. minimum), and $z_{i}, i=1,2,3$, are the three closest non-colineal local minima (resp. maxima)). The $c_{i}$ are defined as [8]

$$
\begin{aligned}
& c_{2}=\frac{\left(x_{l}-x_{1}\right)\left(y_{3}-y_{1}\right)-\left(x_{3}-x_{1}\right)\left(y_{l}-y_{1}\right)}{\left(x_{2}-x_{1}\right)\left(y_{3}-y_{1}\right)-\left(x_{3}-x_{1}\right)\left(y_{2}-y_{1}\right)}, \\
& c_{3}=\frac{\left(x_{2}-x_{1}\right)\left(y_{l}-y_{1}\right)-\left(x_{l}-x_{1}\right)\left(y_{2}-y_{1}\right)}{\left(x_{2}-x_{1}\right)\left(y_{3}-y_{1}\right)-\left(x_{3}-x_{1}\right)\left(y_{2}-y_{1}\right)},
\end{aligned}
$$

$c_{1}=1-c_{2}-c_{3}$.

$L_{i, j} \in \mathbb{R}^{N_{1} \times N_{2}}$ is a discrete approximation of the Laplacian operator which consists of a matrix of the same size as $X$ with all zeros except for the kernel. Here, vec(.) is the classical vectorization operation, in which the columns of a matrix are vertically concatenated to form a vector.

Problem (P-2D) paves the way for $n \mathrm{D}$ extensions, simply by defining the penalization tensor for all local extrema, and a roughening tensor on $n \mathrm{D}$. It also constitutes a true genuine-2D approach. The corresponding 2D algorithm is shown herein. We revealed that such an approach overperforms the existing bidimensional algorithms, namely Image EMD [32], Prox-EMD [48] in its pseudo-2D implementation, and TV-G texture-geometry decomposition [15]. Indeed, UOA-EMD is orientation-independent, shows lower temporal complexity, and performs better in decomposing AM-FM images [8].

In all that follows, and based on previous work [10], $\lambda$ was chosen equal to 1 in the image processing framework.

\section{Results}

\subsection{Comparison of perfusion mean values}

We first compared the mean perfusion value of the neck between the two groups. To this purpose, the mean of the pixel values inside the ROI for each subject was computed in each image, and the average for the 50 images was then determined. The same 


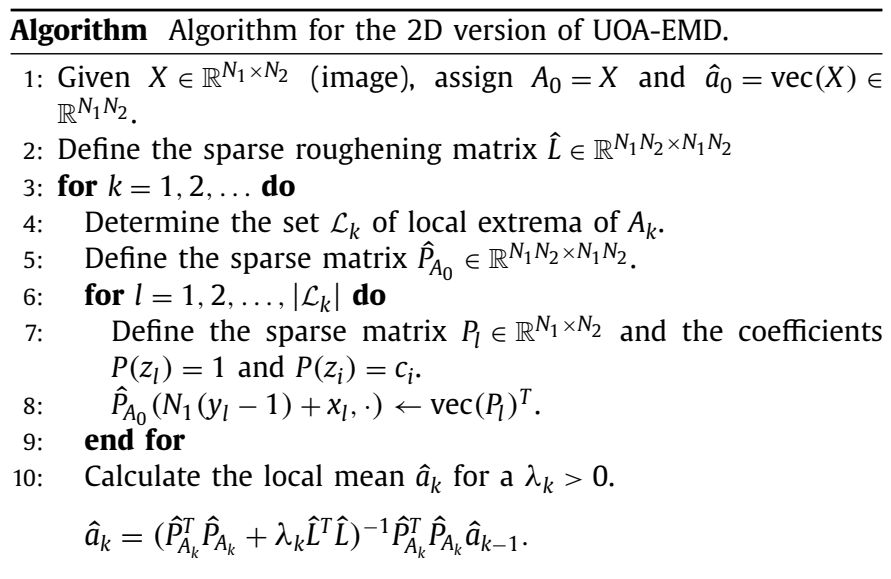

11: Construct $A_{k}$ from $\hat{a}_{k}$ and calculate the mode $D_{k}=A_{k-1}-A_{k}$. 12: end for

Notations: we used the notation $M(n, \cdot)$ to denote the $n$th row of matrix $M$.

Table 1

Detailed results for two subjects: one healthy and one suffering from PXE.

\begin{tabular}{llclll}
\hline Subject & & Perfusion & \multicolumn{2}{l}{ Lacunarity } \\
\cline { 3 - 6 } & & $\left(\right.$ APU $\left._{L S C I}\right)$ & IMF1 & IMF2 & Residue \\
\hline \multirow{2}{*}{ Healthy subject } & Neck & 114.7 & 0.04 & 0.04 & 0.07 \\
& Back & 63.2 & 0.03 & 0.04 & 0.06 \\
PXE patient & Neck & 139.9 & 0.04 & 0.04 & 0.13 \\
& Back & 66.0 & 0.03 & 0.04 & 0.11 \\
\hline
\end{tabular}

Table 2

Statistical results obtained from the Mann-Whitney test between the two groups of subjects (PXE patients and healthy subjects). ns stands for non significantly different between the two groups of subjects. $p<0.05$ corresponds to significant statistical differences.

\begin{tabular}{lll}
\hline & Neck & Back \\
\hline Perfusion & ns & ns \\
Lacunarity for IMF1 & ns & ns \\
Lacunarity for IMF2 & ns & ns \\
Lacunarity for residue & $p=0.0004$ & $p=0.0052$ \\
\hline
\end{tabular}

procedure was performed in ROI of the back. The results show that, in the neck, the mean perfusion value is higher in the PXE group than in the control group (133.7 $\mathrm{APU}_{L S C I}$ in the PXE group and 125.2 $\mathrm{APU}_{L S C I}$ in the control group). The same conclusion is drawn in the back (72.4 $\mathrm{APU}_{L S C I}$ in the PXE group and 64.2 $\mathrm{APU}_{L S C I}$ in the control group). Detailed results from two subjects (one healthy subject and one PXE patient) are presented in Table 1. However, with only 11 subjects in each group, we do not observe any statistical difference between the two groups, neither in the neck, nor in the back (see Table 2). We also note that the standard deviation values are greater in the PXE group, for data recorded in the neck, than in the control group (94.4 APU $\mathrm{ASCI}_{L}$ in the PXE group and 88.0 $\mathrm{APU}_{L S C I}$ in the control group).

\subsection{Patterns revealed by $2 D-U O A-E M D$ on $L S C I$ data}

Figs. 3 and 4 show the results of 2D-UOA-EMD on LSCI data, in the neck and back respectively, both in PXE patients and in healthy subjects. According to these figures we observe that patterns become visible as the number of IMFs increases. The size of these patterns grows with the number of IMFs and is the largest on the residue. This is true both in the images recorded in the neck and in the back, in PXE patients and healthy subjects. We also note that the patterns from the images recorded in the neck are much more visible than those from the images recorded in the back.

\subsection{Quantification of the spatial patterns}

In order to quantify these spatial patterns, we computed the lacunarity of the fractal dimension image of each IMF and residue, in each ROI. We then computed an average value for each subject and for each site [25]. Lacunarity was proposed to differentiate patterns of spatial dispersion [41]: lacunarity is a fractal property that is used to describe the texture of a fractal. More precisely, lacunarity measures the "lumpiness" of the fractal data, providing meta-information about the computed fractal dimension values of the image. The higher the lacunarity, the more inhomogeneous the examined fractal area and vice versa. Lacunarity is defined in terms of the ratio of the variance over the mean value of the function as

$L=\frac{1 /(M N) \sum_{m=0}^{M-1} \sum_{n=0}^{N-1} I(m, n)^{2}}{\left[1 /(M N) \sum_{k=0}^{M-1} \sum_{l=0}^{N-1} I(k, l)\right]^{2}}-1$,

where $M$ and $N$ are the sizes of the fractal dimension image $I$ [40]. In our work, fractal dimension was computed using a differential box counting algorithm [25].

The fractal dimension obtained from each IMF and residue, in both groups and in both sites, is shown in Fig. 5. Moreover, the mean lacunarity values obtained from each IMF and residue, in both groups and in both sites, are shown in Fig. 6. Detailed results from two subjects (one healthy subject and one PXE patient) are presented in Table 1. Figs. 5 and 6 reveal that mean lacunarity (resp. fractal dimension) is higher (resp. lower) in residue than in IMFs, both in the neck and the back. Moreover, mean lacunarity (resp. fractal dimension) is higher (resp. lower) in PXE than in control subjects, in IMFs and residue. This is true both for the images recorded in both the neck and back areas.

The Mann-Whitney test shows that the differences between the lacunarity values computed from the residue in the two groups are statistically significant in the neck $(p=0.0004)$, but also in the back $(p=0.0052)$ (see Table 2). No statistical differences were found for the lacunarity values computed from the IMFs, neither in the neck, nor in the back (see Table 2). The same statistical conclusions were obtained for the fractal dimension (statistical differences for the residue between the two groups, in the neck and in the back).

\section{Discussion}

The skin is the organ that is first affected in PXE and we noticed that the skin in PXE sometimes presents with erythema. This is why we proposed in this document to study skin microvascular perfusion. This was performed through the processing of $\mathrm{LSCl}$ images recorded in the neck and back of healthy subjects and PXE patients. Our image processing framework relies on the recent 2D version of UOA-EMD. We show that the 2D-UOA-EMD is able to reveal hidden patterns in LSCI data reflecting microvascular skin perfusion. These patterns are herein quantified by lacunarity values. From these patterns (in residue) we report statistical differences between patients suffering from PXE and healthy controls, in the back, but most of all in the neck. We also noticed differences in perfusion between the two groups, but no statistical difference was obtained with $11 \times 2$ subjects. The 2 D-UOA-EMD framework is able to underline such differences and reveals that microvascular perfusion is altered in PXE patients. The IMFs computed from the framework are features of the original data and reveal local textures 

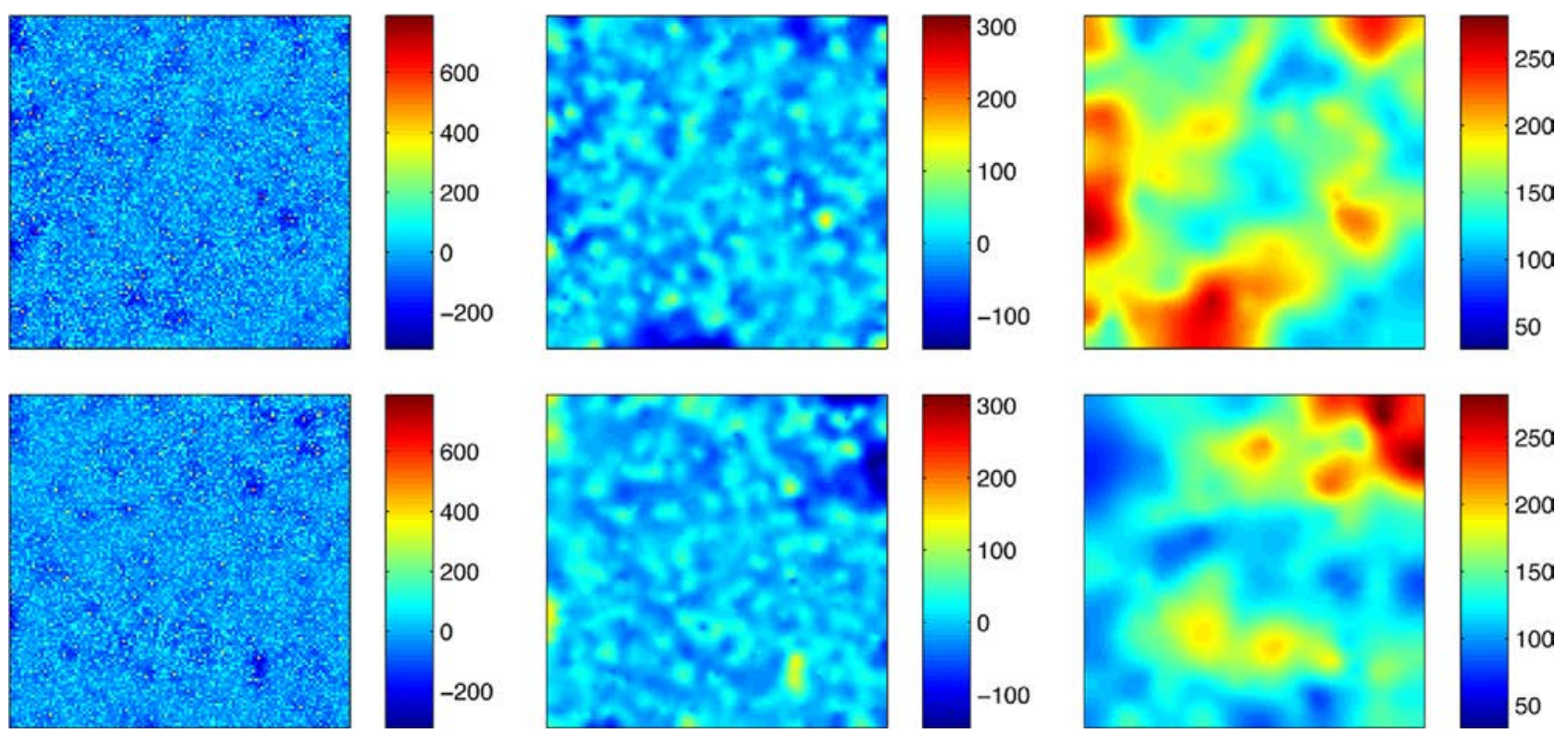

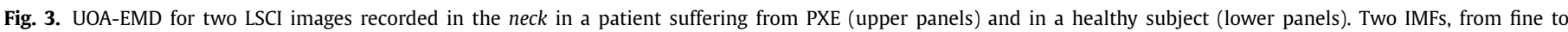

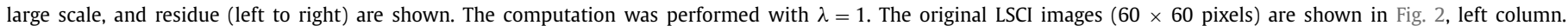
The colorbar unit is $\mathrm{APU}_{L S C I}$ (For interpretation of the references to colour in this figure legend, the reader is referred to the web version of this article.)
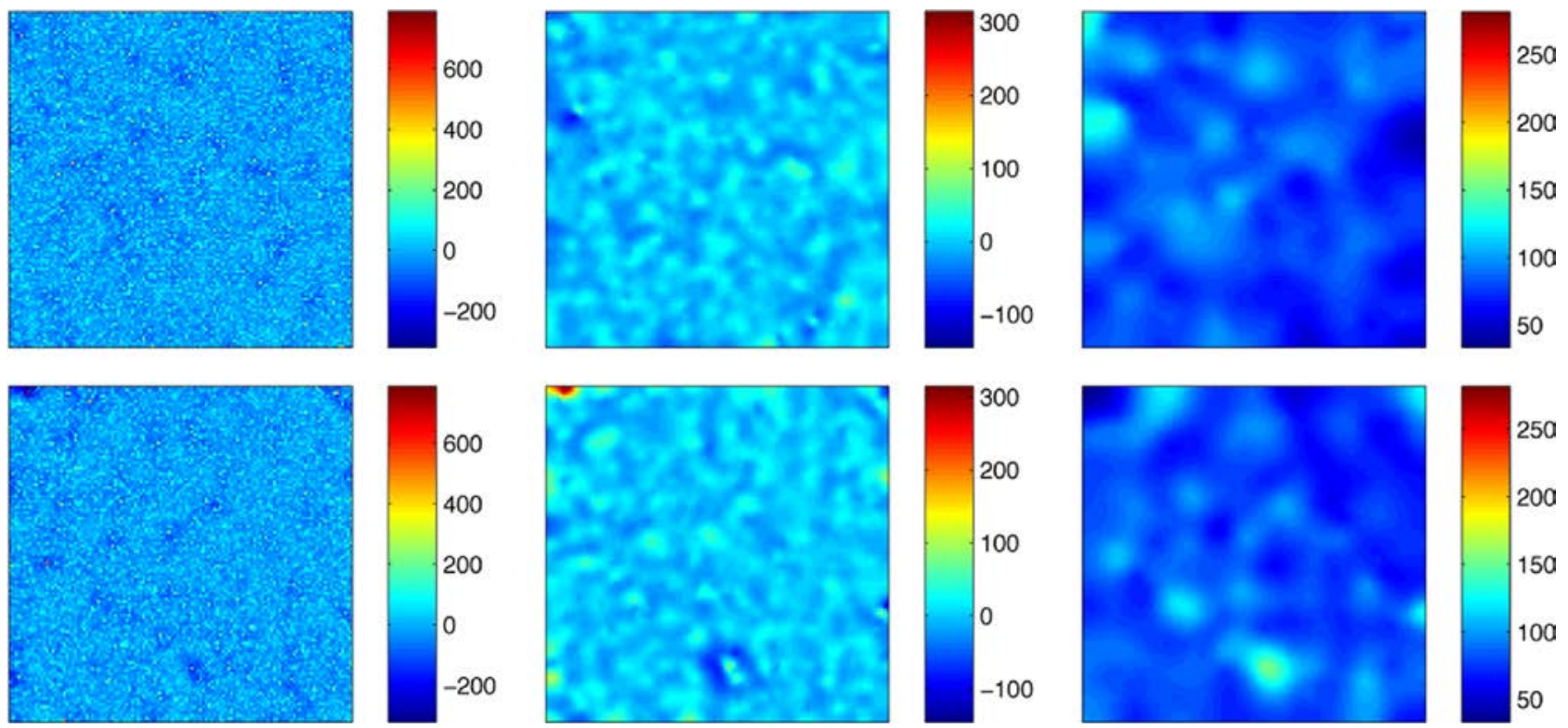

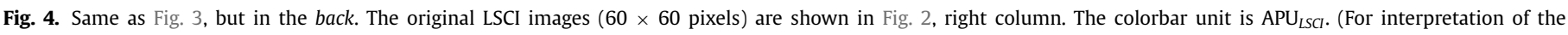
references to colour in this figure legend, the reader is referred to the web version of this article.)

with characteristic spatial frequencies. The first IMF represents the smallest scale (the finest textural), possibly background noise. As the number of IMFs increases, larger scales are discovered. The residue gives only the largest scale of the overall mean trend in intensity of the image. This explains why lacunarity presents larger values in residue than in IMFs. These findings are consistent with another recent study [25].

The Mann-Whitney test shows that the differences between the lacunarity values computed from the residue in the two groups are statistically significant in the neck, but also in the back. However, no statistical differences are found for the lacunarity values computed from the IMFs, neither in the neck, nor in the back. The same statistical conclusions are obtained for the fractal dimension. Since the residue captures higher spatially-changing perfusion trends, these results support the idea that microvascular blood flow is altered in PXE patients compared to control subjects. Moreover, in relation to a previous study [25], our results (higher lacunarity values for residue in PXE patients compared to control subjects) may mean that PXE patients present higher microvascular perfusion than control subjects (in the back but most of all in the neck). The exact physiological link with the pathology remains to be elucidated but the presence of infraclinical inflammatory changes may be hypothesized. As mentioned previously, the cardiovascular system may also be affected in PXE patients. The fact that the lacunarity values for residue are higher in PXE patients than in control subjects not only in the region of the neck (area known as presenting papules in PXE patients) but also in the region of the back (area known as being without papules in PXE patients) may reveal the systemic nature of the condition. 

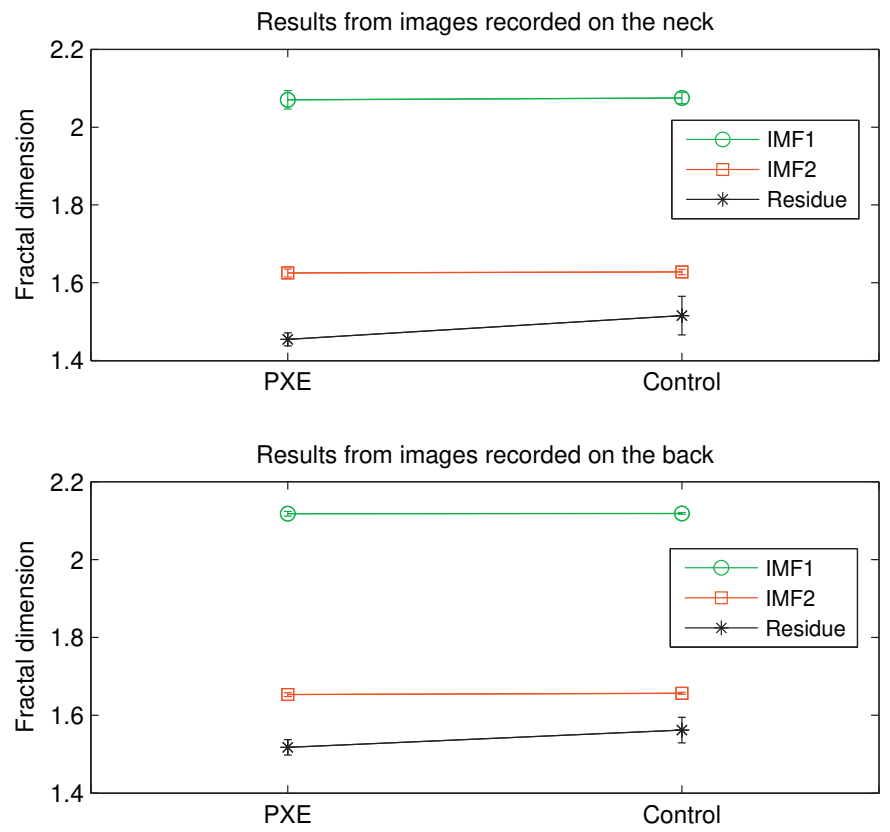

Fig. 5. Mean and standard deviation values for fractal dimension of IMFs and residue obtained with 2D-UOA-EMD. The results were computed from $550 \mathrm{LSCI}$ data recorded in 11 patients suffering from PXE and 550 LSCI data recorded in 11 healthy subjects, at the neck and back; see text for details.
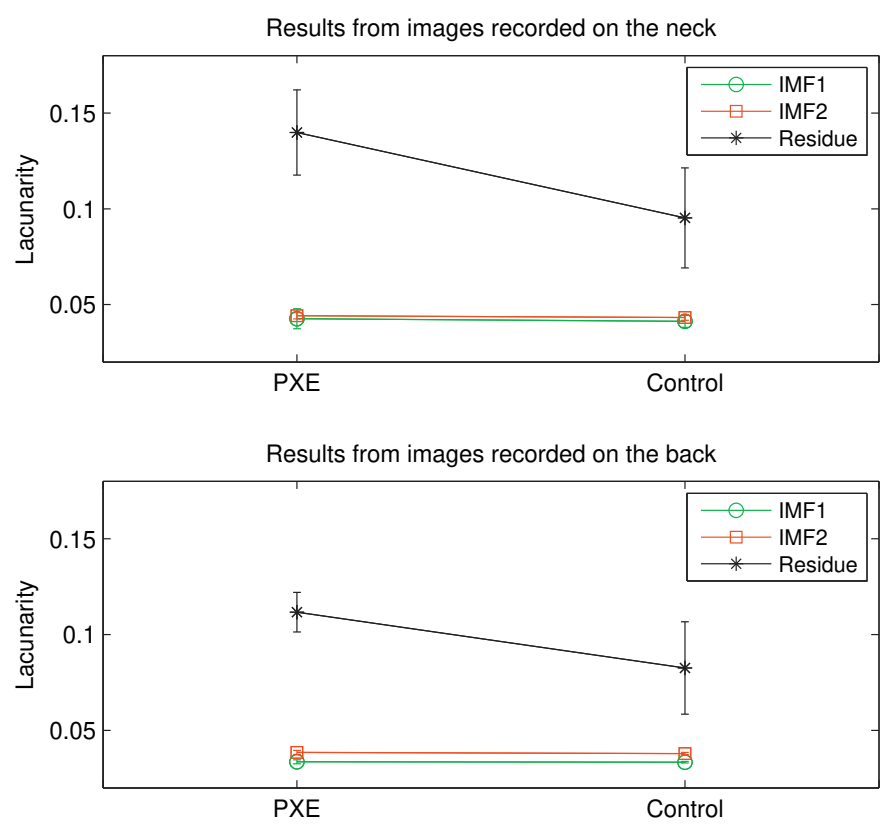

Fig. 6. Same as Fig. 5 but for lacunarity values of fractal dimension images.

Several kinds of algorithms can be used to process images such as LSCI data. Among them, EMD has the advantage of being designed as a fully data-adaptive method [20]: it is fully datadriven and does not need any a priori defined basis system, such as wavelet-based techniques. EMD decomposes non linear and nonstationary data into a set of oscillatory components: IMFs [20]. However, one of the major drawbacks of EMD is the lack of a mathematical framework, even if mathematical foundations have been proposed $[11,12,18,19,37,42,43,51,54]$. This is why Colominas et al. recently reported a new approach for EMD, based on optimization [10]. The advantages of this approach, compared to others, are the following: i) the explicit computation of envelopes to find the local mean is not needed; ii) there is no use of explicit spline interpolation; iii) the proposed method provides an analytical solution. Moreover, the computational cost of this new approach is similar to that of EMD and only one parameter needs to be set [10]. A two-dimensional version of this algorithm has recently been published [8]. By processing synthetic and real images, the superiority of this $2 \mathrm{D}$ version of the unconstrained optimization approach to EMD (2D-UOA-EMD) - compared to other bidimensional versions of EMD-derived algorithms - has been reported [8].

Our work also has some limitations. From a measurement point of view: (i) we processed a small group of subjects (22 subjects). Our results now need to be confirmed using data acquired from a larger population; (ii) the LSCI technique is sensitive to experimental conditions [35]. Therefore, studies such as this necessitate a very well defined measurement protocol; (iii) the data processed in the neck of the subjects may be altered by skin movements. Indeed, LSCI is sensitive to any movement: movement of the skin, voluntary or involuntary movement of the subjects, etc. Since the carotid artery is close to the regions where PXE papules are present in the neck, the pulsed passage of blood into the carotid artery may lead to artefacts in the data recorded in the neck. To avoid this, we could use an adhesive opaque surface applied on the skin $[36,38]$. With reference to image processing: 2DUOA-EMD requires a parameter to be set ( $\lambda$ value). In the present work, the value of $\lambda$ was chosen equal to 1 based on previous work [10]. Further investigation would be necessary to choose the optimal value for $\lambda$.

In the future, other approaches could be used to continue the work. From a medical point of view: with data acquired from many more subjects, we may be able to separate the population into true positive, false positive, true negative, and false negative. Furthermore, it could be interesting to validate the method proposed herein as a noninvasive method to study the possible actions of a future therapy. Moreover, it could be interesting to study how lesions evolve with age, and also compare the results provided by active lesions (lesions that evolve) with those of inactive lesions. With reference to image processing: some variations of 2D-UOA-EMD could be proposed. Thus, for more uniform scales in each mode, noise-assisted variations could be explored. Adding white noise to the image would focus the method on specific scales, from fine scales to coarse scales. Moreover, other multiresolution image processing frameworks could be proposed. Variational mode decomposition (VMD) and its variants could be of interest for this purpose $[13,14,47]$. VMD overcomes the drawback of recursive decomposition algorithms such as EMD: VMD does not require a mode sifting process. All modes are determined simultaneously. This is why VMD is currently drawing increased attention $[27,28,30]$. This algorithm deserves attention in future studies.

\section{Conclusion}

To date no study on skin perfusion in PXE patients has been conducted, in spite of cutaneous lesions noted in the pathology. This is why we proposed an analysis of the local texture of skin perfusion images (LSCI data) in patients suffering from PXE and a comparison of the results with those observed in healthy control subjects. Using a two-dimensional version of the recently proposed UOA-EMD, we have revealed for the first time that microvascular perfusion is altered in PXE patients. These new findings provide valuable information in the study of pathophysiologic changes to the skin in PXE.

\section{Conflict of interest}

The authors declare no conficts of interest. 


\section{References}

[1] J.H. Ahn, D.H. Kwak, B.H. Koh, Fault detection of a roller-bearing system through the EMD of a wavelet denoised signal, Sensors 14 (2014) $15022-15038$.

[2] L. Bercovitch, P. Terry, Pseudoxanthoma elasticum 2004, J. Am. Acad. Dermatol. 51 (2004) S13-S14.

[3] A.A. Bergen, A.S. Plomp, E.J. Schuurman, S. Terry, M. Breuning, H. Dauwerse, J. Swart, M. Kool, S. van Soest, F. Baas, J.B.t. Brink, P.T. de Jong, Mutations in ABCC6 cause pseudoxanthoma elasticum, Nat. Genet. 25 (2000) 228-231.

[4] R. Bi, J. Dong, C.L. Poh, K. Lee, Optical methods for blood perfusion measurement-theoretical comparison among four different modalities, J. Opt. Soc. Am. A 32 (2015) 860-866.

[5] D.A. Boas, A.K. Dunn, Laser speckle contrast imaging in biomedical optics, J. Biomed. Opt. 15 (2010). Article id. 011109

[6] D. Briers, D.D. Duncan, E. Hirst, S.J. Kirkpatrick, M. Larsson, W. Steenbergen, T. Stromberg, O.B. Thompson, Laser speckle contrast imaging: theoretical and practical limitations, J. Biomed. Opt. 18 (2013). Article id. 66018

[7] N. Chassaing, L. Martin, P. Calvas, M.L. Bert, A. Hovnanian, Pseudoxanthoma elasticum: a clinical, pathophysiological and genetic update including 11 novel ABCC6 mutations, J. Med. Genet. 42 (2005) 881-892.

[8] M.A. Colominas, A. Humeau-Heurtier, G. Schlotthauer, Orientation-independent empirical mode decomposition for images based on unconstrained optimization, IEEE Trans. Image Process 25 (2016) 2288-2297.

[9] M.A. Colominas, G. Schlotthauer, M.E. Torres, Improved complete ensemble EMD: a suitable tool for biomedical signal processing, Biomed. Sig. Process. Control 14 (2014) 19-29.

[10] M.A. Colominas, G. Schlotthauer, M.E. Torres, An unconstrained optimization approach to empirical mode decomposition, Dig. Sig. Process. 40 (2015) 164-175.

[11] I. Daubechies, J. Lu, H.T. Wu, Synchrosqueezed wavelet transforms: an empirical mode decomposition-like tool, Appl. Comput. Harmon. Anal. 30 (2011) 243-261.

[12] E. Deléchelle, J. Lemoine, O. Niang, Empirical mode decomposition: an analytical approach for sifting process, IEEE Sig. Process. Lett. 12 (2005) 764-767.

[13] K. Dragomiretskiy, D. Zosso, Variational mode decomposition, IEEE Trans. Sig. Process. 62 (2014) 531-544.

[14] K. Dragomiretskiy, D. Zosso, Two-dimensional variational mode decomposition, in: Energy Minimization Methods in Computer Vision and Pattern Recognition: 10th International Conference, EMMCVPR 2015, hong kong, china, january 13-16, 2015. proceedings, 2015, pp. 197-208.

[15] J. Gilles, S. Osher, Bregman implementation of meyers g-norm for cartoon+ textures decomposition, UCLA Cam Report (2011) 11-73.

[16] S.M. Hacker, F.A. Ramos-Caro, B.B. Beers, F.P. Flowers, Juvenile pseudoxanthoma elasticum: recognition and management, Pediatr. Dermatol. 10 (1993) 19-25.

[17] C. Harrington, F.M. Beck, C.M. Allen, J.R. Kalmar, The prevalence of pseudoxanthoma elasticum-like connective tissue changes in an oral biopsy service and review of the literature, Oral Surg. Oral Med. Oral Pathol. Oral Radiol. 119 (2015) 441-450.

[18] S.D. Hawley, L.E. Atlas, H.J. Chizeck, Some properties of an empirical mode type signal decomposition algorithm, IEEE Sig. Process. Lett. 17 (2010) 24-27.

[19] B. Huang, A. Kunoth, An optimization based empirical mode decomposition scheme, J. Comput. Appl. Math. 240 (2013) 174-183.

[20] N.E. Huang, Z. Shen, S.R. Long, M.C. Wu, H.H. Shih, O. Zheng, N.C. Yen, C.C. Tung, H.H. Liu, The empirical mode decomposition and the hilbert spectrum for nonlinear and non-stationary time series analysis, Proc. R. Soc. London A 454 (1998) 903-995.

[21] A. Humeau-Heurtier, P. Abraham, G. Mahe, Analysis of laser speckle contrast images variability using a novel empirical mode decomposition: comparison of results with laser doppler flowmetry signals variability, IEEE Trans. Med. Imag. 34 (2015) 618-627.

[22] A. Humeau-Heurtier, B. Buard, G. Mahe, P. Abraham, Laser speckle contrast imaging of the skin: interest in processing the perfusion data, Med. Biol. Eng. Comput. 50 (2012) 103-105.

[23] A. Humeau-Heurtier, E. Guerreschi, P. Abraham, G. Mahé, Relevance of laser doppler and laser speckle techniques for assessing vascular function: state of the art and future trends, IEEE Trans. Biomed. Eng. 60 (2013) 659-666.

[24] A. Humeau-Heurtier, G. Mahé, P. Abraham, Microvascular blood flow monitoring with laser speckle contrast imaging using the generalized differences algorithm, Microvasc. Res. 98C (2015) 54-61.

[25] A. Humeau-Heurtier, G. Mahe, P. Abraham, Multi-dimensional complete ensemble empirical mode decomposition with adaptive noise applied to laser speckle contrast images, IEEE Trans. Med. Imag. 34 (2015) 2103-2117.

[26] Q. Jiang, M. Endo, F. Dibra, K. Wang, J. Uitto, Pseudoxanthoma elasticum is a metabolic disease, J. Invest. Dermatol. 129 (2009) 348-354.

[27] Y. Jiang, Z. Li, C. Zhang, C. Hu, Z. Peng, On the bi-dimensional variational decomposition applied to nonstationary vibration signals for rolling bearing crack detection in coal cutters, Meas. Sci. Technol. 27 (2016). Article id. 065103

[28] B. Li, C. Tang, X. Zhu, X. Chen, Y. Su, Y. Cai, A 3d shape retrieval method for orthogonal fringe projection based on a combination of variational image decomposition and variational mode decomposition, Opt. Lasers Eng. 86 (2016) 345-355.

[29] Q. Li, Q. Jiang, E. Pfendner, A. Varadi, J. Uitto, Pseudoxanthoma elasticum: clinical phenotypes, molecular genetics and putative pathomechanisms, Exp. Dermatol. 18 (2009) 1-11.
[30] Z. Li, J. Chen, Y. Zi, J. Pan, Independence-oriented VMD to identify fault feature for wheel set bearing fault diagnosis of high speed locomotive, Mech. Syst. Sig. Process. 85 (2017) 512-529.

[31] J. Lin, Improved ensemble empirical mode decomposition and its applications to gearbox fault signal processing, Int. J. Comput. Sci. 9 (2012) 194-199.

[32] A. Linderhed, Image empirical mode decomposition: a new tool for image processing, Adv. Adapt. Data Anal. 1 (02) (2009) 265-294.

[33] M. Lozano, J.A. Fiz, R. Jane, Automatic differentiation of normal and continuous adventitious respiratory sounds using ensemble empirical mode decomposition and instantaneous frequency, IEEE J. Biomed. Health Inf. 20 (2016) 486-497.

[34] G. Mahe, S. Durand, A. Humeau, G. Leftheriotis, P. Rousseau, P. Abraham, Air movements interfere with laser speckle contrast imaging recordings, Lasers Med. Sci. 27 (2011) 1073-1076.

[35] G. Mahé, S. Durand, A. Humeau-Heurtier, G. Leftheriotis, P. Abraham, Impact of experimental conditions on noncontact laser recordings in microvascular studies, Microcirculation 19 (2012) 669-675.

[36] G. Mahé, P. Rousseau, S. Durand, S. Bricq, G. Leftheriotis, P. Abraham, Laser speckle contrast imaging accurately measures blood flow over moving skin surfaces, Microvasc. Res. 81 (2011) 183-188.

[37] T. Oberlin, S. Meignen, V. Perrier, An alternative formulation for the empirical mode decomposition, IEEE Trans. Sig. Process. 60 (2012) 2236-2246.

[38] L. Omarjee, I. Signolet, A. Humeau-Heutier, L. Martin, D. Henrion, P. Abraham, Optimisation of movement detection and artifact removal during laser speckle contrast imaging, Microvasc. Res. 97 (2015) 75-80

[39] C.D. Papadaniil, L.J. Hadjileontiadis, Efficient heart sound segmentation and extraction using ensemble empirical mode decomposition and kurtosis features, IEEE J. Biomed. Health Inf. 18 (2014) 1138-1152.

[40] M. Petrou, P.G. Sevilla, Image Processing: Dealing with Texture, New York, Wiley, 2006.

[41] R.E. Plotnick, R.H. Gardner, W.W. Hargrove, K. Prestegaard, M. Perlmutter, Lacunarity analysis: a general technique for the analysis of spatial patterns, Phys. Rev. E 53 (1996) 5461-5468.

[42] N. Pustelnik, P. Borgnat, P. Flandrin, Empirical mode decomposition revisited by multicomponent non-smooth convex optimization? Signal Process. 102 (2014) 313-331.

[43] N. Pustelnik, P. Borgnat, P. landrin, A multicomponent proximal algorithm for empirical mode decomposition, in: 20th European Signal Processing Conference, EUSIPCO 2012, 2012, pp. 1880-1884.

[44] L.M. Richards, S.M. Kazmi, J.L. Davis, K.E. Olin, A.K. Dunn, Low-cost laser speckle contrast imaging of blood flow using a webcam, Biomed. Opt. Express 4 (2013) 2269-2283.

[45] F. Ringpfeil, Selected disorders of connective tissue: pseudoxanthoma elasticum, cutis laxa, and lipoid proteinosis, Clin. Dermatol. 23 (2005) 41-46.

[46] M. Roustit, C. Millet, S. Blaise, B. Dufournet, J.L. Cracowski, Excellent reproducibility of laser speckle contrast imaging to assess skin microvascular reactivity, Microvasc. Res. 80 (2010) 505-511.

[47] J. Schmitt, E. Horne, N. Pustelnik, S. Joubaud, P. Odier, An improved variational mode decomposition method for internal waves separation, in: Signal Processing Conference (EUSIPCO), 2015, doi:10.1109/EUSIPCO.2015.7362721. 23rd European.

[48] J. Schmitt, N. Pustelnik, P. Borgnat, P. Flandrin, L. Condat, 2D prony-huang transform: a new tool for $2 \mathrm{~d}$ spectral analysis, IEEE Trans. Image Process. 23 (12) (2014) 5233-5248.

[49] M.E. Torres, M.A. Colominas, G. Schlotthauer, P. Flandrin, A complete ensemble empirical mode decomposition with adaptive noise, IEEE Int. Conf. Acoust. Speech Sig. Process. (ICASSP) (2011) 4144-4147.

[50] A. Utani, M. Tanioka, Y. Yamamoto, R. Taki, E. Araki, H. Tamura, Y. Miyachi Relationship between the distribution of pseudoxanthoma elasticum skin and mucous membrane lesions and cardiovascular involvement, J. Dermatol. 37 (2010) 130-136.

[51] H. Wu, P. Flandrin, I. Daubechies, One or two frequencies? the synchrosqueezing answers, Adv. Adapt. Data Anal. 3 (2011) 29-39.

[52] Z. Wu, N.E. Huang, Ensemble empirical mode decomposition: a noise-assisted data analysis method, Adv. Adapt. Data Anal. 1 (2009) 1-41.

[53] Z. Wu, N.E. Huang, X. Chen, The multi-dimensional ensemble empirical mode decomposition method, Adv. Adapt. Data Anal. 1 (2009) 339-372.

[54] Z. Xu, B. Huang, K. Li, An alternative envelope approach for empirical mode decomposition, Digit. Signal Process. 20 (2010) 77-84

[55] X. Xue, J. Zhou, Y. Xu, W. Zhu, C. Li, An adaptively fast ensemble empirical mode decomposition method and its applications to rolling element bearing fault diagnosis, Mech. Syst. Signal Process. 62-63 (2015) 444-459.

[56] X. Yang, G. Cheng, H. Liu, Improved empirical mode decomposition algorithm of processing complex signal for IOT application, Int. J. Distributed Sensor Net. (2015). Article ID 862807

[57] J.R. Yeh, J.S. Shieh, Complementary ensemble empirical mode decomposition: a novel noise enhanced data analysis method, Adv. Adapt. Data Anal. 2 (2010) 135-156.

[58] Y. Yu, W. Li, D. Sheng, J. Chen, A novel sensor fault diagnosis method based on modified ensemble empirical mode decomposition and probabilistic neural network, Measurement 68 (2015) 328-336.

[59] J. Zheng, J. Cheng, Y. Yang, Partly ensemble empirical mode decomposition: an improved noise-assisted method for eliminating mode mixing, Sig. Process. 96 (2014) 362-374. 